\title{
Structural Stability of a Family of Exponential Polynomial Maps
}

\author{
Francisco Solis ${ }^{1, *} \mathbb{\infty}$, Silvia Jerez ${ }^{1} \mathbb{1}$, Roberto Ku-Carrillo ${ }^{2}$ and Sandra Delgadillo ${ }^{2}$ \\ 1 Center of Research in Mathematics (CIMAT), Guanajuato 36023, Mexico; jerez@cimat.mx \\ 2 Departamento de Matemáticas y Física, Universidad Autónoma de Aguascalientes, Av. Universidad 940, \\ Cd. Universitaria, Aguascalientes 20130, Mexico; raku@correo.uaa.mx (R.K.-C.); \\ sedelgad@correo.uaa.mx (S.D.) \\ * Correspondence: solis@cimat.mx; Tel.: +52-473-7327155
}

Received: 11 March 2020; Accepted: 6 April 2020; Published: 7 April 2020

\begin{abstract}
We perturbed a family of exponential polynomial maps in order to show both analytically and numerically their unpredictable orbit behavior. Due to the analytical form of the iteration functions the family has numerically different behavior than its correspondent analytical one, which is a topic of paramount importance in computer mathematics. We discover an unexpected oscillatory parametrical behavior of the perturbed family.
\end{abstract}

Keywords: perturbed; exponential polynomial; oscillatory parametric behavior

\section{Introduction}

An exponential polynomial function is defined as the sum of products of polynomials and exponential functions [1,2]. Considering these functions as iteration functions, they give rise to exponential discrete dynamical systems which were introduced in [3]. In this work, it was shown that the study of analytical and numerical techniques for such systems are a difficult task. It is important to remark that even theoretical results obtained from these systems often differ from the numerical results obtained by simple evaluation techniques.

In this work, a perturbation to a family of exponential polynomial map is introduced in order to analyze its structural stability. Such family of system models generally emerge from diverse areas from Applied Mathematics such as population models, models for infectious diseases and also from nonstandard discretizations of continuous models [4-9]. Also in Physics, they appear commonly in quantum mechanics as asymptotic solutions to the standard time independent Schrödinger equation [10-12]. Perturbation methods were the main tool to obtain quantitative information from nonlinear models all this before the computational methods revolution. Nowadays, perturbation on mathematical models is often included to add uncertainty and to analyze their response to different factors. For example immigration/emigration in the case of population models or variation of inputs on the level of downstream genes in the case of developmental Biology. In our case, we perturbed a family of exponential polynomial dynamical systems with a constant parameter in order to show as a first goal the differences from its asymptotic behavior when one treats numerically both systems: perturbed and unperturbed. We will show that asymptotic numerical behavior of the perturbed system does not correspond to its asymptotic theoretical behavior by showing different behavior and also displaying oscillating features of the system. We can wonder why to complain and cause a needless commotion about numerical calculations especially when the theory of dynamical systems is available. The fact is that for most exponential polynomial dynamical systems is impossible to apply the basic theory, even to calculate the equilibrium points explicitly. 
Since mathematical modeling is a fast growing area in a variety of areas in Biology and also in new trends of applied mathematics, the new models considered in the literature are becoming more quantitative. Thus, a second goal of this work is to show specialists on those areas the care that one has to consider when analyzing a system using only computational methods. Therefore, we want to emphasize that every computational strategy must be accompanied by a variety of mathematical theories and numerical techniques.

This paper is organized as follows: In Section 2, we introduce the perturbed exponential polynomial family of discrete dynamical systems of our interest and we also show how the perturbation may be considered in Ecology. In Section 3, the analysis of this family is performed by dividing it into two scenarios: the unperturbed case and the perturbed case. Only for the perturbed case we are able to compute explicitly the equilibria and potential periodic points. A stability analysis is carried out in this case. For the perturbed case, we show only an approximation of the equilibria, but we are able to show their asymptotic stability. Next, in Section 4 we discuss numerically the asymptotic behavior of both scenarios and show the reasons for the appearance of the oscillatory behavior. General conclusions of this work are summarized in Section 5.

\section{Exponential Family of Dynamical Systems}

Consider the perturbed family of exponential polynomial discrete dynamical systems given by

$$
x_{n+1}=f\left(\alpha, x_{n}\right)=x_{n}+\left(2-x_{n}\right) e^{-\alpha\left(x_{n}-1\right)^{2}}+\epsilon,
$$

where the parameter $\alpha$ satisfies $\alpha>0$ and $\epsilon$ is a small positive parameter. We assume that $x_{n} \geq 0$; since we may consider that $x_{n}$ has some meaning in the context of several applications, for example in population dynamics. System (1) generalizes a very basic linear discrete dynamical system, known in several applications as exponential growth models $[13,14]$. The new system incorporates a new term that for small values of $\alpha$ can be considered as a small functional perturbation term, which biologically models a period of abundance of resources for the population and for larger values of $\alpha$ such period tends to disappear. The perturbation term will show that the system is not structural stable. Moreover, the perturbation terms can model some biological important quantities as harvesting, immigration, emigration, etc. [15]. Thus, system (1) can be considered as a functional perturbed discrete growth model with an extra term that can be interpreted as a regulatory mechanism of harvesting or migration.

\section{Analysis of the Exponential Polynomial Family}

\subsection{Unperturbed System}

Let us start by analyzing the family of unperturbed systems by finding the equilibrium points and classifying their stability, see [3]. The fixed points of (1) with $\epsilon=0$ satisfy the equation

$$
(2-x) e^{-\alpha(x-1)^{2}}=0 .
$$

Therefore, the only fixed point of the unperturbed system is given by $x=2$, for every value of $\alpha$. That is, $(2, \alpha)$ is a collection of fixed points that is constant for every value of $\alpha$. Since $0 \leq \frac{\partial f}{\partial x}(\alpha, 2)=$ $1-e^{-\alpha}<1$, all fixed points are stable for all non negative values of $\alpha$.

In a typical analysis of a discrete system one will seek for periodic points of period three in order to show that there are periodic points of all orders. In our case we will show that the family does not have periodicity at all. Thus, let us search for periodic points of period two. Such points satisfy:

$$
(2-x) e^{-\alpha(x-1)^{2}}=(2-x)\left(e^{-\alpha(x-1)^{2}}-1\right) e^{-\alpha\left(x+(2-x) e^{-\alpha(x-1)^{2}}\right)},
$$


that is (assuming $x \neq 2$ ),

$$
e^{-\alpha(x-1)^{2}}=\left(e^{-\alpha(x-1)^{2}}-1\right) e^{-\alpha\left(x+(2-x) e^{-\alpha(x-1)}\right)^{2}} .
$$

Setting $z=e^{-\alpha(x-1)^{2}}$, and $\beta=e^{-\alpha\left(x+(2-x) e^{-\alpha(x-1)}\right)^{2}}$, we note that $0<z \leq 1$ and $0<\beta<1$. Substituting these values on (3) we get the equation $z(1-\beta)=-\beta$, which does not have real solutions. Therefore, there are no periodic points of period two implying that there are not periodic points of any period by Sarkovskii's theorem [16]. From the previous analysis, the local asymptotic behavior of the unperturbed system reduces to two cases: its orbits converge to the fixed point or diverge. Now let us find out the behavior of the system numerically. We make use of bifurcation diagrams which are graphical representation of the long-term behavior of the solution as a function of a system's parameter. In our case, such diagrams display properties of the asymptotic solution of a dynamical system as a function of $\alpha$, allowing one to recognize immediately where qualitative changes in the asymptotic solution occur. Let us observe the bifurcation diagrams for the unperturbed system given in Figure 1a for different initial conditions. Here the first 3000 iterations are discarded for each value of $\alpha$. It is important to remark that all calculations were made with a personal computer with an Intel ${ }^{\circledR} \mathrm{Core}^{T M}$ i7-9700 CPU @ $3.00 \mathrm{GHz} \times 8$.

Notice from Figure $1 \mathrm{a}$ that the fixed point $x=2$ seems numerically to be stable only for values of $\alpha$ in $(0,5.56)$. Let us observe that after the value $\alpha=5.56$ the branch of fixed points decreases down to the value of the initial condition. We also obtain the same qualitative behavior when we vary the initial conditions. This behavior was expected since for large values of $\alpha$ the system behaves as $x_{n+1}=x_{n}$ since the basin of attraction of the fixed point disappears for larger values of $\alpha$. Thus, foreshadowing the behavior of the perturbed system.

(a)

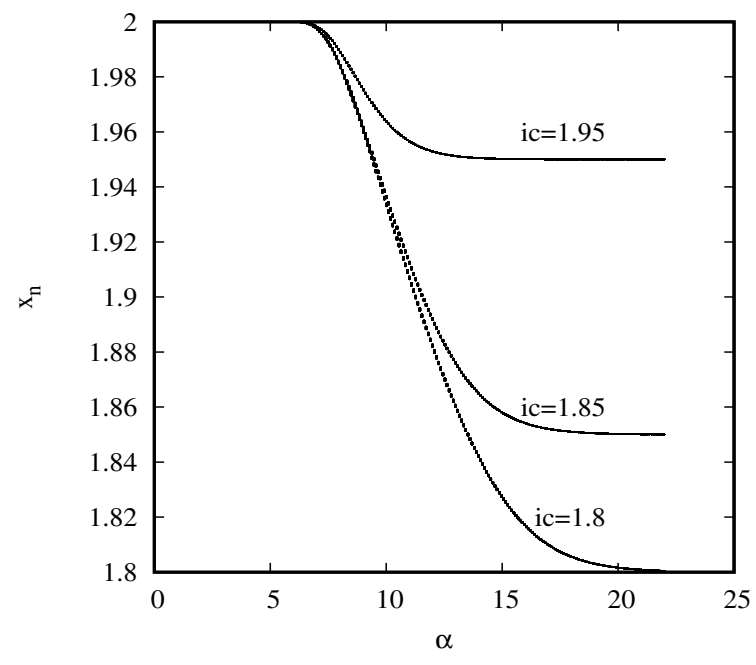

(b)

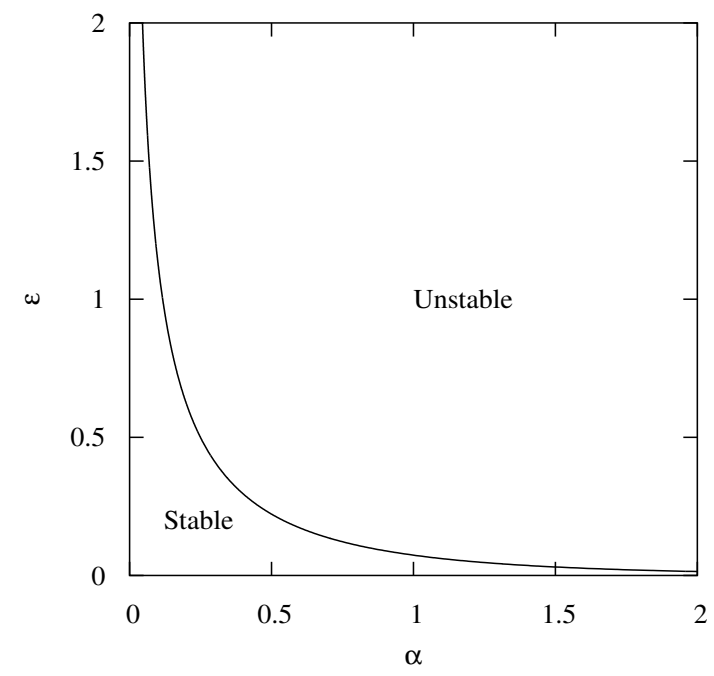

Figure 1. (a) Bifurcation diagram for the unperturbed system (b) Regions of stability and instability for the perturbed system.

\subsection{Perturbed System}

Let us analyze the perturbed system. Its fixed points satisfy:

$$
F(x, \epsilon) \equiv(2-x) e^{-\alpha(x-1)^{2}}+\epsilon=0 .
$$

Using perturbation analysis, we find that the solution of such equation, $x_{\epsilon}$, is given by 


$$
x_{\epsilon}=2+e^{\alpha} \epsilon+2 \alpha e^{2 \alpha} \epsilon^{2}+O\left(\epsilon^{3}\right) .
$$

Since

$$
F^{\prime}\left(x_{\epsilon}, \epsilon\right)=-e^{-\alpha}+4 \alpha \epsilon+(3+2 \alpha) \alpha e^{\alpha} \epsilon^{2}+O\left(\epsilon^{3}\right),
$$

we conclude that such equilibrium point is stable when

$$
0<e^{-\alpha}-4 \alpha \epsilon-(3+2 \alpha) \alpha e^{\alpha} \epsilon^{2}+O\left(\epsilon^{3}\right)<2 .
$$

Here is the first change produced by the perturbation: The equilibrium point loses stability, meaning that now the region of stability is bounded (before was the whole non negative axis). Notice that for very small values of $\epsilon$ the region of stability decreases, see Figure $1 b$.

Let us examine the existence of periodic points of period two. Such potential points must satisfy

$$
(2-x) e^{-a(x-1)^{2}}+\epsilon=\left((2-x)\left(e^{-a(x-1)^{2}}-1\right)+\epsilon\right) e^{-\alpha\left(x+(2-x) e^{-\alpha(x-1)^{2}}+\epsilon-1\right)^{2}}-\epsilon .
$$

The unique solution of this equation is given by the fixed point. Therefore there are not periodic points of period two. Which implies that there are not periodic points of any period. Let us find in the next section how the perturbed system behaves numerically.

\section{Asymptotic Numerical Behavior}

We know by the analysis done previously how the perturbed system must behave asymptotically. Now let us verify those facts numerically. For small values of $\alpha(\alpha \in(0,2.08))$ system orbits converge to the fixed point. So far we have agreement among numerical approximations and theory. Notice that for bigger values of $\alpha(\alpha>2.08)$ the sequence $\left\{x_{n}\right\}_{n=1}^{\infty}$ is increasing. Let us consider the variation of the parameter $\alpha$ with those large values for the perturbed system. For this, we set different small values of $\epsilon$ with different values of the initial condition and discarding the first 3000 iterations keeping the following one hundred iterations. When that finite number of iterations is displayed on the bifurcation diagram, oscillations appear. The resulting bifurcation diagrams show a behavior consisting in a wide collection of oscillatory functions for which the number of waves or bumps seems to depend on the initial condition. Such number of bumps and their amplitudes increases when the value of the initial condition decreases, see Figure 2. It is important to remark that this behavior is not aperiodic or chaotic. What is most important, the system behaves as $x_{n}+\epsilon$ for larger values of $\alpha$. Thus, the oscillatory behavior is preserved. This ghost bump phenomenon is analogous to the Gibbs phenomenon of a Fourier series $[17,18]$. Let us explain briefly this analogy. Gibbs phenomenon is an overshoot of an eigenfunction series approximation occurring at discontinuities of a piecewise continuously differentiable periodic function. Whereas the perturbed system approximates an unknown function with also unknown smoothness properties. Both, the Gibbs phenomenon and the ghost bump phenomenon, reflect the difficulty inherent in approximating numerically a function. The first by a truncated series of a combination of sine and cosine functions and the second by a truncated exponential polynomial which we will discuss next. It is important to remark that numerical simulation methods require stabilization methods when modeling oscillation phenomena. Since numerical overshoot is observed when the error tolerances are too loose and disappear for standard tolerances. Tighter tolerances lead to more accurate results, but increase the computational load. Gibbs phenomenon can can be reduced with a sigma approximation, see [19]. For our case we still do not know if it is possible to stabilize our numerical procedure. 
(a)

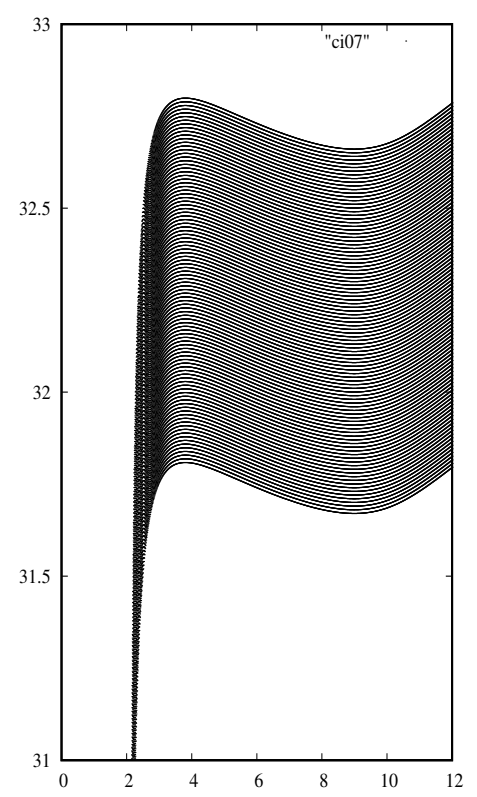

(b)

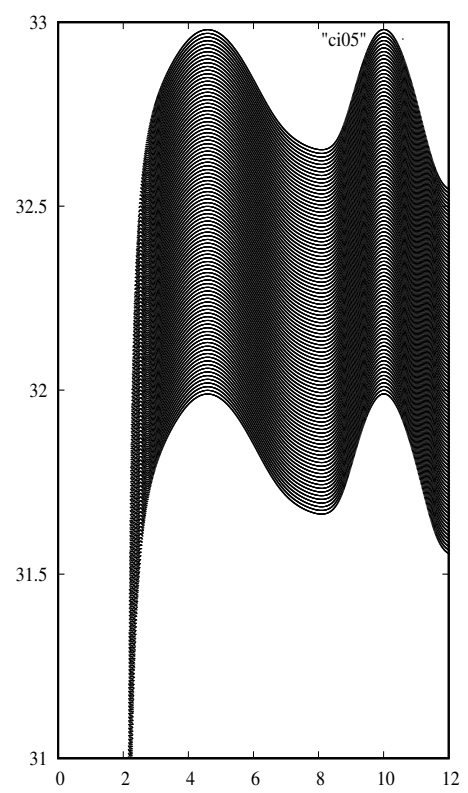

(c)

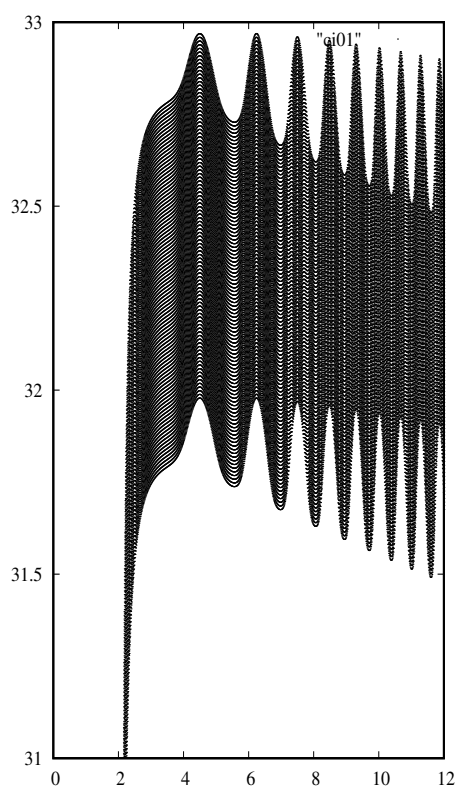

Figure 2. Bifurcation diagram of system (1) with initial conditions (a) 0.7 , (b) 0.5 , (c) 0.1 . Horizontal axis is $\alpha$ and vertical axis is $x_{n}, n=3001-3100$.

Let us show the reasons for the appearance of the oscillatory behavior. The perturbed system can be written as:

$$
x_{n}=x_{0}+\epsilon n+\left(2-x_{0}\right) e^{-\alpha\left(x_{0}-1\right)^{2}}+\sum_{j=1}^{n-1}\left(2-x_{j}\right) e^{-\alpha\left(x_{j}-1\right)^{2}} .
$$

Each term in the sum contributes to generate a new bump in the graph of the function $x_{n}=x_{n}(\alpha)$. Thus, For fixed $n$, the graph of the function $x_{n}(\alpha)$ will have $n-1$ bumps and then it will decay exponentially, with $\alpha$ as rate of decay, to the value $x_{0}+n \epsilon$. Notice that the terms in the sum drop off quickly enough so the new iteration will inherit the previous bumps.

Regarding the variation of the initial condition, let us notice that the graph of $x_{n}(\alpha)$ for fixed $n$ and fixed initial condition is compressed from the right, as compared with the graph for the same $n$ but with a smaller initial condition. Therefore, the initial condition indicates how frequently the function oscillates, playing a similar role as the angular frequency for harmonic oscillators [20].

It is important to remark that the behavior shown for system (1), is shared by many discrete exponential polynomial systems. Thus, an open question rises if such families act with accordance to a set of universal properties which are independent of the specific exponential polynomial considered. We should like briefly to illustrate this fact by way of the following example. Let us only modify the coefficients on the perturbed system in order to get a similar asymptotic behavior as system (1). Let us replace the term $(x-1)^{2}$ by the term $(x-c)^{2}$ in the system (where $c$ is a constant). This modification makes the amplitude of the oscillations changed considerably. Moreover, the oscillation only appears when the constant $c$ is less than 2 and for larger values of the parameter, see Figure 3a. Finally, if we replace now the term $2-x$ by the term $d-x$ in the perturbed system (where $d$ is a constant) the fixed point now becomes $x_{f}(\epsilon)=d-e^{\alpha(d-1)} \epsilon+\mathcal{O}\left(\epsilon^{2}\right)$, which is stable for $d \lesssim 5 / 2+1 / 2(\sqrt{1 / a-41 / 2})$. This new system also presents oscillations for most values of $d$, see Figure $3 \mathrm{~b}$. Therefore, we conclude that the exponential polynomial is the main cause of oscillations. 
(a)

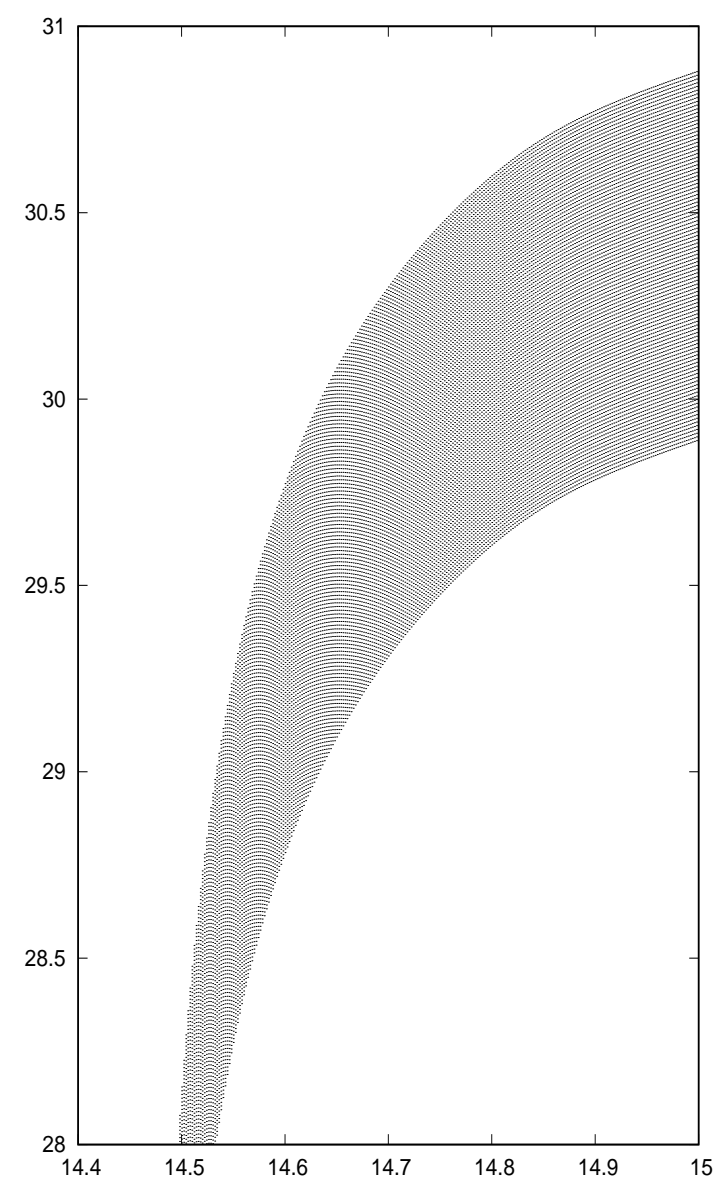

(b)

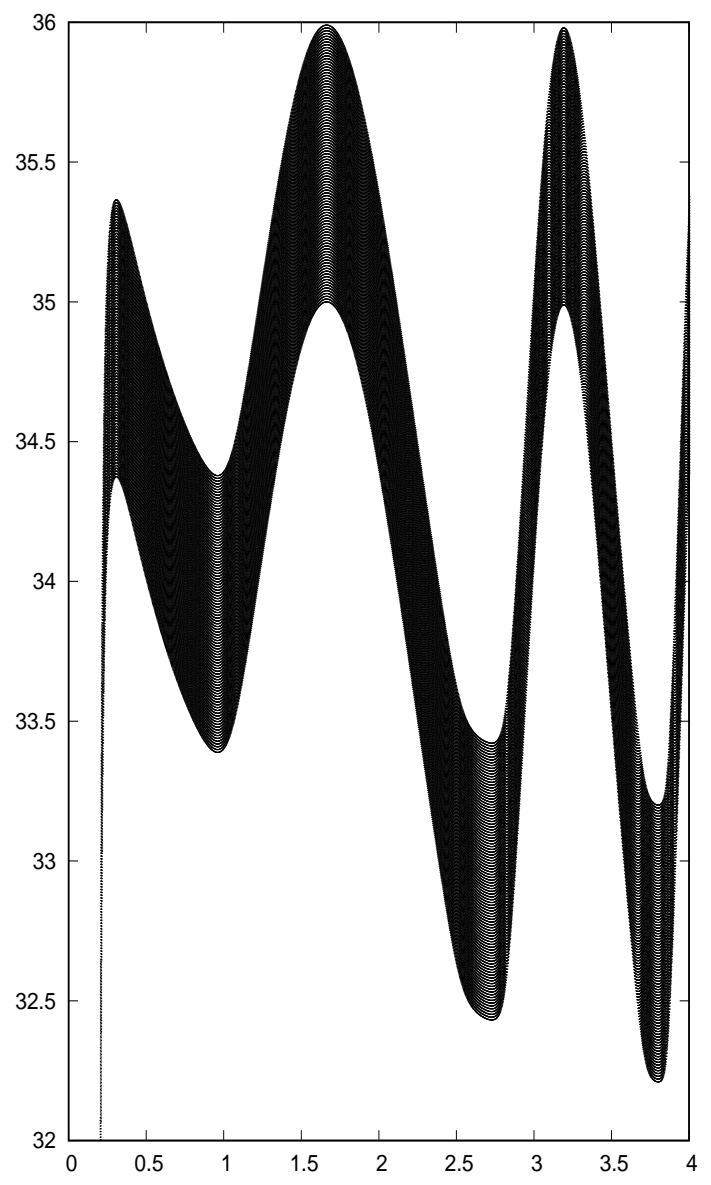

Figure 3. Bifurcation diagrams of modified system (1) with (a) $c=1.7,(\mathbf{b}) d=5$ an initial condition $x_{0}=0.017$. Horizontal axis is $\alpha$ and vertical axis is $x_{n}, n=3001-3100$.

\section{Conclusions}

The study and analysis of exponential polynomial discrete dynamical systems is a new trend in Applied Computational Mathematics. One of the fundamental properties of a system is its structural stability, which means that the qualitative behavior of its trajectories is unaffected by small perturbations. In this work we have shown that a family of these system does not posses such a property even for constant perturbations. Our methodology used for this research was to show numerically and analytically the loss of stability of the unique fixed point for the perturbed system. We have discovered and established that polynomial discrete systems are complicated to analyze and most of the times even the equilibria are impossible to be provided with explicit closed-forms. This fact makes hard to implement standard analysis techniques. In essence we relied on computational methods to analyse the family of interest. We have shown that these systems exhibit transient behavior that remained for large number of iterations which does not correspond to their asymptotic behavior. A characteristic that may led to wrong results if one is using only numerical calculations due to the impossibility of carry out an analytical process. In the end, the determinative fact that the iteration function is a combination of products of exponential functions and polynomials no matter what the degree are on the given polynomials, a given exponential function will eventually decay faster than the polynomials.

One of the unpleasant features when considering only a finite number of iterations is the appearance of numerical oscillations. We showed the main reasons for the appearance of such oscillatory behavior. So far we are not able to verified if the computer numerical truncation is another factor. 
In summary, our analysis of the perturbed exponential family is just a starting point to develop a more general theory for general polynomial discrete dynamical systems. We have discovered that are new open research venues such as potential stabilization methods which is a future research project.

Author Contributions: Formal analysis, F.S., S.J., R.K.-C. and S.D.; Methodology, F.S.; Writing一original draft, F.S., S.J. and S.D.; Writing-review \& editing, F.S. and R.K.-C. All authors have read and agreed to the published version of the manuscript.

Acknowledgments: This work was supported by CONACYT project CB2016-286437.

Conflicts of Interest: The authors declare that they have no conflict of interest.

\section{References}

1. Bell, E.T. Exponential polynomials. Ann. Math. 1934, 35, 258-277. [CrossRef]

2. Dickinson, D.; Warsi, S.A. On a generalized Hermite polynomial and a problem of Carlitz. Bollettino dell'Unione Matematica Italiana 1963, 18, 256-259.

3. Solis, F. Evolution of an exponential polynomial family of discrete dynamical systems. Math. Comput. Appl. 2019, 24. [CrossRef]

4. Arenas, A.J.; González-Parra, G.; Chen-Charpentier, B.M. A nonstandard numerical scheme of predictor-corrector type for epidemic models. Comput. Math. Appl. 2010, 59, 3740-3749. [CrossRef]

5. Arenas, A.J.; Moraño, J.A.; Cortés, J.C. Non-standard numerical method for a mathematical model of RSV epidemiological transmission. Comput. Math. Appl. 2008, 56, 670-678. [CrossRef]

6. Wood, D.T.; Kojouharov, H.V.; Dimitrov, D.T. Universal approaches to approximate biological systems with nonstandard finite difference methods. Math. Comput. Simulat. 2017, 133, 337-350. [CrossRef]

7. Wood, D.T.; Kojouharov, H.V. A class of nonstandard numerical methods for autonomous dynamical systems. Appl. Math. Lett. 2015, 50, 78-82. [CrossRef]

8. Jódar, L.; Villanueva, R.J.; Arenas, A.J.; González, G.C. Nonstandard numerical methods for a mathematical model for influenza disease. Math. Comput. Simulat. 2008, 79, 622-633. [CrossRef]

9. Villanueva, R.J.; Arenas, A.J.; González, G.C. A nonstandard dynamically consistent numerical scheme applied to obesity dynamics. J. Appl. Math. 2008. [CrossRef]

10. Dirac, P.A.M. The Principles of Quantum Mechanics; Oxford University Press: Oxford, UK, 1958.

11. Zhu, W.; Huang, Y.; Kouri, D.J.; Arnold, M.; Hoffman, D.K. Time-dependent wave-packet forms of Schrödinger and Lippmann-Schwinger equations. Phys. Rev. Lett. 1994, 72, 1310-1313. [CrossRef] [PubMed]

12. Surján, P.R.; Ángyán, J. Perturbation theory for nonlinear time-independent Schrödinger equations. Phys. Rev. A 1983, 28, 45-48. [CrossRef]

13. Imhof, L.A. Maximin designs for exponential growth models and heteroscedastic polynomial models. Ann. Stat. 2001, 29, 561-576. [CrossRef]

14. Tsoularis, A.; Wallace, J. Analysis of logistic growth models. Math. Biosci. 2002, 179, 21-55. [CrossRef]

15. Clutton-Brock, T.H.; Coulson, T.N.; Milner-Gulland, E.J.; Thomson, D.; Armstrong, H.M. Sex differences in emigration and mortality affect optimal management of deer populations. Nature 2002, 415, 633-637. [CrossRef] [PubMed]

16. Baldwin, S. Generalizations of a theorem of Sarkovskii on orbits of continuous real-valued functions. Discrete Math. 1987, 67, 111-127. [CrossRef]

17. Wilbraham, H. On a certain periodic function. Camb. Dublin Math. J. 1848, 3, 198-201.

18. Hewitt, E.; Hewitt, R.E. The Gibbs-Wilbraham phenomenon: An episode in Fourier analysis. Arch. Hist. Exact Sci. 1979, 21, 129-160. [CrossRef]

19. Burger, W.; Burge, M.J. Principles of Digital Image Processing: Core Algorithms; Springer: Berlin, Germany, 2009; pp. 231-232.

20. Giancoli, D.C. Physics for Scientists and Engineers; Pearson Education International: London, UK, 2008.

(C) 2020 by the authors. Licensee MDPI, Basel, Switzerland. This article is an open access article distributed under the terms and conditions of the Creative Commons Attribution (CC BY) license (http:/ / creativecommons.org/licenses/by/4.0/). 